\title{
Pharmacological management of dermatomyositis
}

\section{Zoltán Griger, Melinda Nagy-Vincze \& Katalin Dankó}

To cite this article: Zoltán Griger, Melinda Nagy-Vincze \& Katalin Dankó (2017):

Pharmacological management of dermatomyositis, Expert Review of Clinical Pharmacology, DOI: 10.1080/17512433.2017.1353910

To link to this article: http://dx.doi.org/10.1080/17512433.2017.1353910

Accepted author version posted online: 08 Jul 2017.

Submit your article to this journal ๔

Q View related articles $\widetilde{ }$

View Crossmark data \lceil 
Publisher: Taylor \& Francis

Journal: Expert Review of Clinical Pharmacology

DOI: $10.1080 / 17512433.2017 .1353910$

Review

\section{Pharmacological management of dermatomyositis}

Zoltán Griger $^{1}$, Melinda Nagy-Vincze ${ }^{1}$, Katalin Dankó ${ }^{1}$

${ }^{1}$ University of Debrecen, Faculty of Medicine, Division of Clinical Immunology;

Corresponding author:

Katalin Dankó

4032 Debrecen, Móricz Zs. krt. 22. Hungary

Tel: +36-52-411-717/54254

Fax: $+36-52-255-218$

email: katalin.danko@gmail.com

\section{Abstract}

Introduction: Dermatomyositis is a rare heterogeneous systemic autoimmune disease with multiple organ involvement which can result in significant disability and mortality. Despite the lack of placebocontrolled trials, glucocorticoids are considered to be the mainstay of initial management. Treatment strategies are mainly based on uncontrolled studies, evidence based guidelines for treatments do not exist.

Areas covered: This review provides an overview of the currently available pharmacological treatments in the field of dermatomyositis including conventional immunosuppressants, biologics and topical agents. The role of antibodies in different treatment responses of dermatomyositis related clinicoserological syndromes is also discussed. A PubMed search was performed in order to find relevant literature for this review.

Expert commentary: Early recognition and intervention is essential to ameliorate disease outcome. Determination of antibodies provide a useful key in diagnosis, clinical manifestations, malignancy, prognosis, and treatment response and may lead to wider acceptance of personalized medicine. 
Corticosteroids with adjunctive steroid-sparing immunosuppressive therapies are recommended to treat disease activity, prevent mortality, and reduce long-term disability. Combinations of secondline therapies or newer third-line therapies are used in severe, refractory, or corticosteroiddependent diseases. Further research is required to assess the role of new therapies.

Keywords: antibodies, anti-MDA5, anti-Mi2, antisynthetase syndrome, anti-TIF1, azathioprine, cyclophosphamide, cyclosporine-A, dermatomyositis; intravenous immunoglobuline, rituximab, methotrexate, mycophenolate mofetyl, tacrolimus 


\section{Abbreviations:}

ACR: American College of Rheumatology

ARS: anti-aminoacyl tRNA synthetases

ASS: antisynthetase syndrome

AZA: Azathioprin

CADM: clinically amyopathic dermatomyositis

CDASI: cutaneous dermatomyositis disease area and severity index

CK: creatine kinase

$\mathrm{Cl}$ : Confidence interval

CNI: calcineurin inhibitor

CTD: connective tissue disease

CYC: cyclophosphamide,

CSA: cyclosporine-A

EULAR: European League Against Rheumatism

FDA: Food and Drug Administration

DM: dermatomyositis

DMARD: disease modifying anti-rheumatic drugs

DOI: definition of improvement

GC: glucocorticoids

HAQ: Health Assessment Questionnaire

HMGCR: 3-hydroxy-3-methylglutaryl-coenzyme A reductase

HR: hazard ratio

HRCT: High Resolution Computer Tomography

IBM: inclusion body myositis

IIM: idiopathic inflammatory myopathies

ILD: Interstitial lung disease

IMACS: International Myositis Assessment and Clinical Studies Group

IMNM: immune-mediated necrotizing myopathy

IV: intravenous

IVIG: intravenous immunoglobuline

JDM: juvenile dermatomyositis 
MAA: myositis-associated antibodies

MDA5: anti-melanoma differentiation associated gene 5

MDAAT: Myositis Disease Activity Assessment Tool

MMT: manual muscle testing

MSA: and myositis-specific antibodies

MMF: mycophenolate mofetyl,

MTX: methotrexate

NXP2: anti-nuclear matrix protein 2

PFT: pulmonary function test

PM: polymyositis

PRINTO: Pediatric Rheumatology International Trials Organization

RIM: Rituximab in Myositis Trial

RP-ILD: rapidly progressive interstitial lung disease

RTX: rituximab

SAE: anti-small ubiquitin-like modifier activating enzyme

SCIG: subcutaneous immunoglobulin

TAC: tacrolimus

TIF1 $\gamma$ : anti-transcription intermediary factor 1 gamma

TIS: total improvement score

TNF: Tumor necrosis factor

VAS: visual analogue scale 
1. Introduction:

Dermatomyositis (DM) is a subtype of idiopathic inflammatory myopathies (IIM), collectively known as myositis and encompasses a group of rare heterogeneous systemic connective tissue diseases. Based on different clinical and histopathological features, IIM can be classified as polymyositis (PM), dermatomyositis, immune-mediated necrotizing myopathy (IMNM) and inclusion body myositis (IBM). According to the Bohan and Peter Criteria [1], the diagnosis of DM is based on a combination of certain clinical and laboratory features, such as (1) the presence of skeletal muscle weakness; (2) elevated serum levels of muscle enzymes; (3) myopathic triad on electromyography; (4) characteristic histopathological changes on muscle biopsy; and (5) the presence of characteristic skin rashes, including the heliotrope rash or Gottron papules over the joint extensor surfaces.

Myositis syndromes are the main causes of acquired muscle diseases in adults, but IIMs are still rare diseases. The annual incidence of IIM is 2-10 per 1 million and overall prevalence is $50-100$ cases/million inhabitants depending on the data origin [2-5]. The portion of DM among IIM in adults is ranging between 26-83\%, depending on genetic, geographic and environmental factors [5-7]. Dermatomyositis is the predominant form of myositis seen in children (80\%-85\% of cases) [8-9].

Dermatomyositis is a multisystem disorder with a wide variety of clinical manifestations including lung, joint, esophageal and cardiac findings; however its hallmark features are the characteristic skin manifestations and progressive symmetrical muscle weakness. In some adult and juvenile cases, presentation and disease course is variable with different manifestations. Some have severe muscle disease at onset, whereas some are amyopathic. Some are more at risk of lung disease or inflammatory arthritis, in contrast to those who have more skin involvement with complications including ulceration or calcinosis. Treatment responses are also variable in these patients, depending on disease phenotype and autoantibody presence. Autoantibodies can be found in specific markers for myositis, and schematically can be categorized as myositis-associated antibodies (MAAs) and myositis-specific antibodies (MSAs). Around $70 \%$ of adult patients and $60 \%$ of juvenile patients now have well-defined autoantibody specificities. An increasing number of MSAs and their corresponding autoantigen targets have been identified. MSAs define patients into clinical phenotypes and potentially can be used as prognostic and therapeutic markers [10-11].

Survival ranges from $75 \%$ to $90 \%$ in patients with adult dermatomyositis and is greater than 95\% in those with juvenile dermatomyositis [12-13]. The low incidence of DM and the nonavailability of validated assessment and improvement criteria of the disease severely hampered the conduct of controlled drug studies in the past. Therefore treatment strategies were based on clinical observations from case series, rather than randomized trials and remained largely consensus driven. Moreover, the heterogeneity, different extramuscular involvement of the disease, and features of clinicoserological syndromes affect the survival and treatment response of the individual.

This review focuses on (1) the different pharmacological agents used in the treatment of DM and (2) the different treatment responses of dermatomyositis related clinicoserological syndromes. 
Body:

\section{Treatment strategy:}

Until now there have not been any therapies approved for the treatment of DM by the Food and Drug Administration or the European Medicines Agency based on randomized controlled trials. The reason behind this is the lack of validated clinically meaningful outcomes. In order to adequately assess the efficacy of therapy, first of all reliable, validated outcome measures are required. As DM is a complex, multisystem disorder, there is no single gold-standard measure for assessing disease activity. The International Myositis Assessment and Clinical Studies (IMACS) group has developed a consensus on a set of Core Set Domains and Measures for the assessment of disease activity, disease damage and patient-reported outcomes in adult and juvenile myositis patients [14]. Disease activity assesses the manifestations of myositis which are thought to be reversible and result directly from the inflammatory process, whereas disease damage is thought to be those persistent changes in anatomy, physiology, pathology or function which result from previously active disease and from complications of therapy or other events [15]. The IMACS Disease Activity core set measures are the followings: (1) physician's global disease activity assessment by visual analogue scale (VAS); (2) patient/parent's global disease activity assessment on VAS; (3) muscle strength measured by manual muscle testing (MMT); (4) physical function measured by Health Assessment Questionnaire (HAQ); (5) serum levels of muscle enzymes; (6) extra muscular activity: Myositis Disease Activity Assessment Tool (MDAAT). The use of these measures is recommended in all myositis therapeutic trials and clinical studies, instructions and educational resources are available and the measures have been validated by IMACS [15].

The recent development of American College of Rheumatology/European League Against Rheumatism (ACR/EULAR) criteria for minimal, moderate, and major clinical response in adult dermatomyositis and polymyositis marks a major advancement in assessing response in myositis clinical trials and studies. These response criteria are sensitive and specific and provide a way to determine clinically meaningful change corresponding to degree of clinical improvement [16-17]. A total improvement score (range 0-100), determined by summing scores for each core set measure, was based on improvement in and relative weight of each core set measure. Thresholds for minimal, moderate, and major improvement were $\geq 20, \geq 40$, and $\geq 60$ points in the total improvement score (TIS) [16-17].

The primary goal of therapy is to increase muscle strength, facilitate the management of daily activity, treat systemic involvements, and skin rashes, prevent joint contractures and the improve the quality of life. The core therapeutic approach remains oral corticosteroid therapy, along with adjunctive steroid-sparing immunosuppressive therapies, which are used to treat disease activity, prevent mortality, and attempt to reduce long-term disability.

3. Overall management

3.1 Glucocorticoids: 
The first line therapy of dermatomyositis is glucocorticoid administration. It is widely accepted that it improves survival, muscle strength, normalizes muscle enzyme levels, reduces disease activity, and disease related disability [18], however no placebo controlled trial with glucocorticoids was carried out. The initial dose of prednisone or it's equivalent recommended as $0.5-1 \mathrm{mg} / \mathrm{kg} / \mathrm{day}$, for at least 4 weeks and the dose should be tapered monthly by $10-20 \%$ to the minimal effective dose based on the improvement of muscle function. When the daily prednisone dose reaches 5-10 $\mathrm{mg} /$ day, the tapering is frequently held for total duration of therapy from 6 to 12 months to prevent relapse. Several factors could be contributed to disease response, including disease subtype, antibody status and malignancy. In a retrospective cohort study, none of the patients with a long delay to diagnosis (greater than 18 months) responded completely, compared with $34 \%$ of those with a short delay (less than 3 months)[18]. Patients with severe disease manifestations such as marked weakness, severe dysphagia or rapidly progressive interstitial lung disease require pulse intravenous methylprednisolone (1000 mg daily for three consecutive days) followed by the highdose oral glucocorticoid regimen [19]. These patients generally should be started on a steroid-sparing immunosuppressive drug.

\subsection{DMARDs}

Conventional immunosuppressive drugs (disease modifying anti-rheumatic drugs), such as methotrexate, azathioprine, cyclosporine-A are considered to be a first line therapies besides steroid in patients with myositis. These have steroid sparing effect, also can decrease the steroid related side effects, and can treat the extramuscular manifestations.

There are no prospective, blinded, controlled studies of methotrexate (MTX) in dermatomyositis, but limited number of case series indicated that MTX might be a useful drug in DM. In patients with JDM, the Pediatric Rheumatology International Trials Organization (PRINTO) study showed that MTX is the first line used immunosuppressive drug worldwide [20]. In one retrospective study MTX led to a significant reduction of cutaneous dermatomyositis disease area and severity index (CDASI). In contrast, the lymphocytic infiltrate in primary skin lesions of MTX responders was significantly more pronounced than that in nonresponders [21]. In a retrospective study, response to MTX seemed to be better in men than in women [18]. There was a study investigating the safety and efficacy of combined MTX + glucocorticoids (GC) treatment compared to GC alone (Prometheus). Twenty-seven patients completed, the primary endpoint was the total dose of glucocorticoids administered between baseline and the end of treatment, the results were not yet reported [22]. In a retrospective study, the 5-year survival was similar among patients treated with MTX and those treated with AZA, whereas survival was higher in the MTX group from 5 years onward [23]. In contrast, in a Chinese survival analysis study of patients with dermatomyositis showing use of azathioprine had better survival [24]. The presence of lung disease might influence the selection of immunosuppressive agents, with AZA potentially being chosen for ILD patients to avoid MTX-associated lung injury. In addition, although the FDA considered azathioprine a class D drug, there are substantial data supporting its safety in pregnancy, whereas MTX is contraindicated [25]. Combination therapy with methotrexate and azathioprine could be also considered when patients have failed to respond to 
either agent alone, as demonstrated in a randomized, crossover study of 30 patients with refractory IIM [26].

T cells play important role in the pathogenesis of dermatomyositis, thus targeting $T$ cells with calcineurin inhibitors, such as tacrolimus (TAC) and cyclosporine-A (CSA) provides an excellent therapeutic option in IIM. In a systematic review of 8 non-randomized, mainly retrospective study the tacrolimus doses of $0.075 \mathrm{mg} / \mathrm{kg} / \mathrm{day}$ or $1-3.5 \mathrm{mg} /$ day were given and were administered twice daily or once a day. According to the comprehensive analysis of the studies, $93.3 \%$ (42/45) and 64.7 $\%(11 / 17)$ of patients showed improvement in muscle strength and physical function status [27], In a more recent retrospective study of 66 patients with PM $(n=28)$ and DM $(n=38)$ found that concomitant use of systemic tacrolimus with GC provides a favorable outcome, lower frequency of relapse and longer periods of remission $(P=0.0001, P=0.001$, respectively) [28]. In a trial comparing the effect of cyclosporine-A versus MTX showed no significant differences between the two treatment arms, but administration of MTX or CSA to corticosteroids was associated with clinical and laboratory improvement [29]. One small retrospective study of patients with DM and acute/subacute ILD ( $n=16)$ found that initial administration of CSA and prednisolone at the early stage of ILD have better outcome in survival rate than prednisolone alone [29]. The same group from Japan found that early intervention with prednisolone and CSA improved the findings of PFTs and chest HRCT scans and that the level of CSA was significantly correlated with the improvement of PFT findings in patients with DM and acute/subacute ILD $(n=14)$ [31]. The favorable effect of intravenous use of CSA was demonstrated in another Japanese study in severe DM cases [32].

Cyclophosphamide (CYC) is an alkylating agent, which is used mainly in patients with DM and rapidly progressing ILD and/or vasculitis. Although CYC in PM/DM with interstitial pneumonia produced inconsistent results in a few case reports, an open-label study of 17 patients has found that, monthly administered IV CYC in different doses and durations improved symptoms, pulmonary function tests and HRCT findings [33]. It can be also used in those patients, who develop flare of ILD after GC and CSA treatment [32], and in children with severe and refractory JDM, where CYC appeared to have provided major clinical benefit with no evidence of serious toxicity in the short term [34]. However potentially severe side effects, such as neutropenia, infections, irreversible ovarian failure leading to infertility and late malignancy could occur after treatment, the optimal dose and duration of the treatment is not defined. Recently, the use of IV CYC according to the EuroLupus Nephritis protocol showed improvement of PFT-s and HRCT findings without any adverse events or drug toxicity [35].

Mycophenolate mofetil (MMF) inhibits $\mathrm{T}$ and $\mathrm{B}$ cell proliferation via impeding the guanosine nucleotide synthesis. There are no prospective large randomized trials and the results of small case series are conflicting. In a study, 10 patients with idiopathic dermatomyositis were treated with mycophenolate mofetil in combination with corticosteroids. Successful steroid taper without disease relapse was achieved in six patients, but in three patients, treatment was associated with opportunistic infections, leading to death in one patient [36]. Another retrospective study found MMF therapy useful in patients $(n=4)$ with DM and ILD [37] and in DM with severe skin manifestations [38].

3.3 Intravenous immunoglobulin (IVIG) 
In addition to its use for the treatment of primary and secondary immunodeficiencies, IVIG is increasingly used for immunomodulating therapy in the treatment of patients with a variety of autoimmune and inflammatory neurological disorders. However, there is only 1 placebo-controlled clinical study in 15 subjects with refractory DM published so far, employing a dose of $2.0 \mathrm{~g} / \mathrm{kg}$ IVIG or placebo for 12 weeks. After a 1-month washout phase the subjects crossed over to the alternate therapy [39]. Subjects on IVIG had a significant improvement in muscle strength and neuromuscular symptoms in contrast to subjects on placebo. A total of 12 subjects received IVIG of which 9 subjects had a major improvement to nearly normal function. In another retrospective study, thirteen patients treated with IVIG ( $2 \mathrm{~g} / \mathrm{kg}$, every 4 weeks) for refractory cutaneous dermatomyositis and all patient improved [40]. A prospective Japanese trial examined the efficacy of $2 \mathrm{~g} / \mathrm{kg}$ IVIG once in steroid refractory IIM and have found that efficacy rate was 93,3\% (14/15 patient) as assessed using MMT [41]. Another open label study found that IVIG as add on treatment with MMF is effective in severe and refractory myositis; all the 7 patients (4 PM and 3 DM) achieved complete remission, determined by increase of muscle strength and normal CK levels [42]. The use of IVIG was also studied in JDM patients. In a large retrospective study 30 steroid refractory patients received IVIG and were compared to 48 similar matched controls. IVIG-treated patients maintained similar or lower disease activity, despite having greater disease activity at baseline [43].

Recently, subcutaneous immunoglobulin ( $\mathrm{SCIG}$ ) has been demonstrated to be a valuable alternative to IVIG in primary immunodeficiencies. Some advantages linked to SCIG are a more favorable side effect profile and better sustained serum IgG levels. A prospective open-label singlecentre study demonstrated significant improvement in muscle enzymes, muscle weakness, quality of life, after SCIG administration in seven patients with active and refractory inflammatory myopathies [44]. In addition, administration of SCIG was beneficial and safe in maintaining a quiescent disease and in inducing a complete remission in moderately active disease in patients with DM or PM [45]. Nowadays a number of studies are registered to prove the efficacy of IVIG/SCIG in IIM, one of them is prospective, double-blind, randomized, placebo-controlled (NCT02728752).

\subsection{Rituximab:}

Rituximab (RTX) is an anti-CD20 monoclonal antibody targeting B cells, which has been successfully used in the treatment of several autoimmune diseases. B cells play a critical role in the initiation and propagation of the immune response and are specifically implicated in the pathogenesis of myositis [46]. Usage of rituximab in refractory IIMs has been reported in several small case reports and case series [47]. A small open-label uncontrolled study showed that all evaluable patients with dermatomyositis exhibited major clinical improvement, with increasing muscle strength [48]. The Rituximab in Myositis (RIM) was the largest study performed in connection with inflammatory myopathies [49]. Two hundred patients were enrolled (76 with PM, 76 with DM, and 48 with juvenile DM), who had failed response with steroid and another immunosuppressive agent. Patients were randomized to receive either rituximab earlyer or rituximab 8 weeks later, and glucocorticoid or immunosuppressive therapy was also allowed at study entry. The primary end point was to compare the time to achieve the IMACS preliminary definition of improvement (DOI) between the 2 groups. The final results did not show difference in the time to improvement (20.2 or 20.0 weeks), however, $83 \%$ of the randomized refractory patients met the DOI (median time was 20 weeks). Rituximab was generally well tolerated and safe and it demonstrated a significant steroid- 
sparing effect over the period of the study (mean daily prednisone dose decreased from $20.8 \mathrm{mg}$ at baseline to $14.4 \mathrm{mg}$ at the end of the clinical trial). Post hoc analysis showed that presence of an antisynthetase, primarily anti-Jo-1, anti-Mi-2, or other autoantibody predicted a shorter time to improvement compared to the absence of autoantibodies, and moreover JDM subtype versus adult also predicted improvement [50]. The autoantibody levels in myositis subjects (Anti-Jo-1, anti-TIF1-g and anti-Mi-2) decreased after $\mathrm{B}$ cell depletion and were correlated with changes in disease activity [51], which argue for the important role of these autoantibodies in the disease pathogenesis.

\subsection{Other biologics}

Abatacept is a fusion protein between immunoglobulin and the extracellular domain of cytotoxic T-lymphocyte antigen 4 that exerts an anti-inflammatory effect by down- regulating T-cell activation. A case report of a recalcitrant JDM complicated by ulceration and calcinosis found beneficial effect of abatacept [52]. Later a randomized, treatment delayed-start trial was performed to assess the effects of abatacept on disease activity and on muscle biopsy features of adult patients with PM and DM. Among 20 randomized patients, 17 were included in the analyses and $8(47 \%)$ achieved the DOI after 6 months of active treatment [53]

Tumor necrosis factor (TNF)-a may play a role in the pathogenesis of DM [54]. In a pilot study, assessing the safety and tolerability of etanercept in dermatomyositis, results showed significant difference between the treatment groups favoring etanercept in the time from randomization to the first occurrence of treatment failure, but rash worsened in 5 etanercept-treated subjects [55]. In addition, etanercept also showed a steroid-sparing effect (median prednisone dose after 24 weeks in the etanercept-treated group 24 was 1.2 vs. $29.2 \mathrm{mg} /$ day; $\mathrm{P}=0.02$ ), but only 16 patients were randomized, 11 patients received etanercept. In another trial of patients with refractory juvenile DM, etanercept did not demonstrate appreciable improvement and some patients noted worsening of disease [56]. In addition, several case reports have been published to demonstrate a TNF inhibitor induced dermatomyositis [57-58]. As a conclusion, these agents should be considered when other therapies are ineffective.

\subsection{Topical agents:}

Topical agents could be useful supplementary treatments in diseases, where skin involvement is dominant. Ultraviolet radiation intensity was associated with the relative proportion of patients with dermatomyositis and with the proportion of patients expressing anti-Mi-2 autoantibodies in the United States [7]. The cutaneous manifestations are extremely photosensitive, thus patients should be educated on the importance of UVA, UVB, and sun protection factor levels, topical sunscreens are commonly used and recommended. Topical steroids are largely used in combination with oral treatments. For control of erythema and pruritus associated with inflammatory disease, topical corticosteroid treatment is usually necessary, although this should not be used on the face or intertriginous areas [59]. Corticosteroid-impregnated tape or topical corticosteroids covered with plastic food wrap have been recommended for treatment of refractory or hyperkeratotic lesions [60]. Usage of topical calcineurin inhibitors are also reported, amyopathic dermatomyositis and classical dermatomyositis patients without muscle disease activity had success with topical $0.1 \%$ tacrolimus [61-62]. Cases of two DM patients showed beneficial effect of topical pimecrolimus, which may be a 
therapeutic alternative for the management of the cutaneous lesions of DM [63]. Respecting the safety profile of topical calcineurin inhibitors, its use should be considered in both children and adults with cutaneous manifestations of dermatomyositis refractory to other treatments. In order to use these options as an alternative or adjunctive therapy, however, further randomized controlled trials are needed.

\section{Dermatomyositis related clinicoserological syndromes:}

Autoantibodies are believed to have a key role in the pathogenesis of myositis and have been identified in over $50 \%$ of patients with IIM. It has been demonstrated that these autoantibodies target both nuclear and cytoplasmic components of the cell and they have traditionally been divided into two subsets, myositis-associated autoantibodies (MAAs) and myositis-specific autoantibodies (MSAs). These antibodies have been extensively demonstrated to correlate with specific clinical manifestations, they are important biomarkers for myositis, aiding in diagnosis and helping to classify patients into more homogeneous groups [64]. The antibodies which are associated with dermatomyositis and skin involvements are the followings: anti-aminoacyl tRNA synthetases (ARS), anti-Mi2, anti-SAE, anti-MDA5, anti-NXP2, and anti-TIF1gamma [65].

\subsection{Antisynthetase syndrome:}

Anti-ARS autoantibodies are the most frequently detected antibodies in adult patients with myositis. According to recent research anti-ARS autoantibodies include anti-Jo-1, anti-PL-7, anti-PL12, anti-OJ, anti-EJ, anti-KS, anti-SC, anti-JS, anti-Ha and anti-Zo autoantibodies [66]. The presence of these antibodies define a distinct clinical phenotype, antisynthetase syndrome (ASS), which is characterized by poor prognosis and multiple organ involvement, such as myositis, ILD, non-erosive arthritis, Raynaud's phenomenon, mechanic's hand, skin rashes, and fever. ILD is by far the most severe manifestation of ASS, occurs in around $80 \%$ of ASS patients, and is associated with high morbidity and mortality. A metaanalysis comparing anti-Jo-1-positive and non- Jo-1 ARS-positive patients reported an increased likelihood of myositis, arthralgia and mechanics hands in anti-Jo-1positive patients, with non-Jo-1 ARS-positive patients having an increased frequency of DM skin lesions, fever and ILD [66]. Furthermore, anti-Jo-1-positive patients have been shown to have a better 5- and 10-year survival compared to non-Jo-1 ARS-positive patients [67-68]. It is important to consider non-JO-1 ARS in patients presenting with ILD and non-descript CTD symptoms in the absence of obvious myositis, even when screening autoantibody tests are negative. The commercial availability of more accurate testing and an increased awareness of these patients among rheumatologists and pulmonary specialists will likely lead to better patient outcomes and more thoughtful treatment strategies [68].

Survival of patients is mainly determined by the presence or absence of ILD: In a cross sectional analysis of 831 patients from a single center revealed that the risk of death was statistically significantly higher among participants with ILD compared to those without ILD (HR 2.13. $95 \% \mathrm{Cl}$ 1.06-4.25; $p=0.03$ ) [69]. The coexistence of anti-SS-A and anti-Jo-1 antibody may be a good predictor for a more coarse and severe ILD in IIM patients who require a more aggressive therapy [70]. The aim of management in this population of patients is to reverse disease progression. The pharmacological therapy contains immunosuppression; typically including oral immunomodulatory agents, IV methylprednisolone and IV CYC. In refractory cases decisions to initiate B cell depletion with 
rituximab are implemented. RTX seems to be more effective in antisynthetase patients [50, 71, 72], thus it is a valuable option in refractory cases.

\subsection{Mi-2}

The frequency of anti-Mi-2 is $9-24 \%$ in adult IIM and $4-10 \%$ in JDM [64]. Clinically, anti-Mi-2 is associated with "classic dermatomyositis", a range of cutaneous features including Gottron's papules, heliotrope rash, V-sign and shawl sign rashes, and cuticular overgrowth [73-76]. These patients have more favorable prognosis, milder muscle involvement and a decreased risk of ILD and malignancy. Patients with anti-Mi-2 autoantibodies tend to respond well to immunosuppressive therapy. In therapy refractor cases RTX seems to be effective. The RIM study demonstrated that the presence of anti-Mi-2 autoantibodies predicted a shorter time to improvement compared to the absence of these autoantibodies after RTX administration (hazards ratio 2.5, $\mathrm{P}<0.01$ ) [50].

\subsection{TIF1gamma}

The target of TIF1Y as a $155 \mathrm{kDa}$ protein with a further $140 \mathrm{kDa}$ band was identified transcription intermediary factor 1 gamma [77]. The frequency of anti-TIF1Y is $13-21 \%$ in adult and $23-29 \%$ in juvenile IIM cases [73]. Clinical representation of patients with anti- TIF1Y is DM associated with malignancy in adult patients. A meta-analysis reported a sensitivity of anti TIF1 $1 \gamma$ for diagnosing cancer-associated DM was $78 \%$ (95\% Cl $45-94 \%$ ), and specificity was $89 \%$ (95\% Cl $82-93 \%)$. The diagnostic OR was 27.26 (95\% Cl 6.59-112.82) [78]. In juvenile DM patients, anti-TIF-1Y antibodies are not associated with malignancy, but rather, with skin ulcerations and more extensive cutaneous diseases, without calcinosis [73]. Similarly, calcinosis was negatively associated with TIF1 $y$ antibodies in adult patients [79]. In the RIM study, anti-TIF1Y levels were decreased after RTX administration and correlated with changes in disease activity [51].

\subsection{MDA5}

Anti-MDA5 autoantibodies target melanoma differentiation associated gene 5 (MDA5), a cytoplasmic protein, which were originally found in an Asian adult DM cohort [80]. The occurrence of the antibody differs in the world. The overall frequency of anti-MDA5 antibodies was significantly higher in Chinese patients than in Japanese cohort ( $36.6 \%$ versus $15.8 \%, P<0.001$ ) [81]. In contrast, the frequency is substantially lower in the USA and in European cohorts, only being described in 0$13 \%$ of DM patients [64]. Patients from the East Asian population are reported to have clinically amyopathic dermatomyositis (CADM), rapidly progressive ILD (RP-ILD) and poor prognosis [73], Chinese tend to have classic DM [81]. Patients from the USA with anti-MDA5 positivity are reported to have mild muscle symptoms and DM, which is associated with ulcers; arthritis and ILD. The phenotype is frequently a clinical mimic of the antisynthetase syndrome and is not associated with rapidly progressive ILD [82]. Ferritin is a useful predictive marker for RP-ILD, and serum ferritin levels predict the development and severity of RP-ILD in PM/DM patients and correlate with disease activity [83]. The therapeutic strategy in RP-ILD patients with hyperferritinemia and/or anti-MDA5 positivity might be a combination therapy of corticosteroids, IV CYC, and calcineurin inhibitors (CNIs), such as CSA and TAC, and should be immediately administered [83]. However, approximately 
$20 \%-40 \%$ of RP-ILD with anti-MDA5 patients die within 6 months after diagnosis, even if a combination therapy of corticosteroids, IV CYC, and CNIs is immediately administered [81, 84]. RTX or MMF administration may be considered when RP-ILD is refractory to the therapies described above [83].

\subsection{SAE}

This antigen is the small ubiquitin-like modifier activating enzyme (SAE). It is almost exclusively present in DM with a frequency of 6-8\%. Patients with anti-SAE had a high frequency of cutaneous lesions including heliotrope and Gottron rash and often develop dysphagia [85-86]. The presence of ILD is not common and the prognosis is good [87].

\subsection{NXP2}

Anti-NXP2 autoantibodies target a $140-k D a$ autoantigen which known as nuclear matrix protein 2 (NXP2). The frequency of this antibody is 1,6-23\% [86; 88-89]. Similarly to anti-TIF1Y positivity, the adult and juvenile patients also have different phenotypes when this antibody is present. Calcinosis is frequently found in both form, but the disease course is severe in adult patients and malignancy might be typical, whereas in juvenile cases, malignancy is not frequent. [86; 88-89]. Stratification by sex revealed that anti-NXP-2 was specifically associated with cancer in males [90], in addition to this intensive cancer search should be performed as part of the diagnostic procedure. Treatment strategy in patients with calcinosis is not known. However, different calcinosis-specific treatments could be administered, such as calcium-channel blockers, bisphosphonates, warfarin, IVIG, colchicine, rituximab, infliximab, sodium thiosulphate, but none of these treatments allowed the reduction of calcinosis or the prevention of new sites involved [91].

\subsection{HMGCR}

Autoantibodies recognizing 3-hydroxy-3-methylglutaryl-coenzyme A reductase (HMGCR) are found in adult myositis patients with immune-mediated necrotizing myopathy (IMNM) and prior statin exposure. In a screen of 750 adult myositis patients' sera no patients had typical DM rashes [92]. In contrast, in a cohort of 440 juvenile patients, five were positive and three of them had rashes characteristic of juvenile dermatomyositis [93]. In another juvenile cohort, four from 381 patients were anti-HMGCR positive, and two of these patients had cutaneous disease [94]. None of these patients had prior statin medication. The presence of the antibody was associated with severe disease and high CK levels. 


\section{Conclusion}

We can agree based on the prior literary summery that thanks to the lack of controlled trials, current therapies used in this disease are mainly based on anecdotes and uncontrolled studies. The core therapeutic approach remains oral corticosteroid administration, along with adjunctive steroidsparing immunosuppressive agents, such as MTX, azathioprine, CSA and MMF. Determination of autoantibodies in the sera of patients with DM is an important key to define disease subtypes, assess clinicoserological classification, and it can also help in the decision making to find the best treatment option. In refractory cases administration of IVIG, or calcineurin inhibitors might be effective. Rituximab is also a valuable option, thus $83 \%$ of the patients improved in the RIM trial. Multicenter, randomized trials, international collaborations, widespread useage of registries and validated outcome measures are required to further facilitate the development of optimal therapeutic alternatives in dermatomyositis. 


\section{Expert Commentary}

There are many fields in the area of pharmacological treatment of dermatomyositis where unmet need could be found. The efficacy of currently available medical treatments are mainly based on clinical observations on case series and not on randomized, placebo controlled trials. Thus there are currently no therapies approved by the Food and Drug Administration or the European Medicines Agency. Nevertheless there are many newly available therapies, demonstrating outstanding results in distinct disease subtypes. On the other hand there are special scenarios, where the available management has poor outcomes, such as calcinosis, or refractory RP-ILD. One key weakness of the clinical management is that the diagnosis of the disease have substantial delay in certain cases and this leads to irreversible changes in the tissues and results as an incomplete response, despite the best treatment regimen. Furthermore, after recognition and diagnosis of the disease, the diagnostic procedure should contain the investigation of every potential (extramuscular) organs, which could be affected. Detection of recently identified autoantibodies might provide major advantages in the diagnosis, organ involvement, complications, prognosis and treatment response, however many of them have poor availability and require specialist facilities and training and is unsuitable for use in most routine diagnostic laboratories. In addition, these antibodies are currently not involved in the classification criteria, which might be changed in the future based on the development and validation of IMACS International Myositis Classification Criteria Project. Based on these arguments, we strongly recommend the treatment and follow up of DM patients in centers with qualified, experienced specialists.

The ultimate goal of future research is to find better drugs, which provide a curative solution for the patients, if it is possible. To achieve this, intensive collaborations are required between clinical and basic science. The exact etiology and pathogenesis of the disease is still unclear. Better understanding of the pathomechanism might lead to construction certain molecules which could target the immune/non-immune cells, cytokines, transduction, or adhesion molecules, resulting sustained remission/cure of the disease. Well designed, prospective trials employing validated standardized outcome measures are required to develop an evidence-based approach to the treatment of dermatomyositis. One big challenge for future development is the heterogeneity and rarity of the disease. Because of that significant progress for an individual researcher at a single center could be slow and inadequately powered, hampered by a lack of adequate patient information and specimens. To skip this challenge, clinical registries and biorepositories, especially international, global registries have proven extremely useful. If clinical data are linked to biospecimens, including serum, DNA, or tissue biopsy samples, they become powerful tools to explore and define disease pathogeneses, biomarkers, and genetic and environmental risk factors [95]. The EuroMyositis register [96] is an international collaboration, which has been created in order to obtain uniform, longitudinal data over adult and juvenile myositis cases to achieve increased knowledge on disease course and prognosis of myositis. The register contains demographic data, laboratory parameters, diagnostic results, disease activity and damage data according to the IMACS core set measures. Information from treatment and longitudinal data of the disease course in combination with research purposes might help to face the abovementioned challenges.

There are some encouraging research projects with molecules targeting key pathogenetic pathways, including interferon signature, lymphocyte recirculation, T-cell interactions, toll-like 
receptors, complement components, which may hold promise but require further investigation in myositis. 


\section{Five year view}

There are some promising preliminary data, which argue for that in five years from this point, new, validated classification criteria will be used for diagnosing inflammatory myopathies. Results from well powered ongoing studies using recently developed clinical response criteria might have potential for approval of a drug for the treatment of myositis. Better availability and further development of imaging (MRI, PET), and serum biomarkers will possess a greater impact in diagnosis and treatment response. With more widespread useage of international registries and IMACS consensus-derived disease activity measures will provide a major advancement understanding disease pathogeneses and will define the optimal therapy in the future.

8. Key issues

- The primary goal of pharmacological therapy is to normalize muscle strength, prevent joint contractures, treat systemic involvements and skin rashes and to improvement the quality of life.

- Corticosteroids are still the first-line treatment of the disease with combination of conventional immunosuppression, such as MTX, azathioprine, or cyclosporine-A.

- Determination of myositis specific antibodies provides a useful key in diagnosis, clinical manifestations, malignancy, prognosis and treatment response in dermatomyositis patients.

- Early detection and treatment of life-threatening organ involvement is crucial to decrease mortality

- Rituximab and IVIG has good results in refractory cases, with certain autoantibody positivity, but there is a need for larger randomized controlled trials.

\section{Funding}

This paper was not funded.

\section{Declaration of Interest}

The authors have no relevant affiliations or financial involvement with any organization or entity with a financial interest in or financial conflict with the subject matter or materials discussed in the manuscript. This includes employment, consultancies, honoraria, stock ownership or options, expert testimony, grants or patents received or pending, or royalties.

\section{Acknowledgements}

The authors would like to thank Dr. Beáta Kovács for handling language and grammar editing. 
References:

* of importance

** of considerable importance

1. Bohan A, Peter JB. Polymyositis and dermatomyositis (first of two parts). N Engl J Med. 1975;292 (7):344-347.

2. Oddis CV, Conte CG, Steen VD, et al. Incidence of polymyositis-dermatomyositis. J Rheumatol. 1990;17(10):1329-1334.

3. Vargas-Leguás, H., Selva-O'Callaghan, A., Campins-Martí, M., et al. Polymyositisdermatomyositis: incidence in Spain (1997- 2004). Med. Clin. (Barc), 2007, 129(19), 721-724

4. Kuo, C. F., See, L. C., Yu, et al. Incidence, cancer risk and mortality of dermatomyositis and polymyositis in Taiwan: a nationwide population study. Br. J. Dermatol., 2011, 165(6), 12731279.

5. Nagy-Vincze M, Bodoki L, Griger Z, et al. Epidemiology of idiopathic inflammatory myopathy in Hungary. Orv Hetil. 2014 Oct 12;155(41):1643-6.

6. Okada S, Weatherhead E, Targoff IN et al. Global surface ultraviolet radiation intensity may modulate the clinical and immunologic expression of autoimmune muscle disease. Arthritis Rheum 2003, 48:2285-2293

7. Love LA, Weinberg CR, McConnaughey DR et al. Ultraviolet radiation intensity predicts the relative distribution of dermatomyositis and anti-Mi-2 autoantibodies in women. Arthritis Rheum. 2009 August ; 60(8): 2499-2504

8. Feldman BM, Rider LG, Reed AM, et al. Juvenile dermatomyositis and other idiopathic inflammatory myopathies of childhood. Lancet. 2008; 371(9631):2201-2212

9. Wedderburn LR, Rider LG. Juvenile dermatomyositis. Best Pract Res Clin Rheumatol. 2009;23(5):665- 678

10. Gunawardena H, Betteridge ZE, McHugh NJ. Myositis specific autoantibodies: their clinical and pathogenic significance in disease expression. Rheumatology (Oxford). 2009 Jun;48(6):607-12.

11. Tansley S, Gunawardena H. The evolving spectrum of polymyositis and dermatomyositismoving towards clinicoserological syndromes: a critical review. Clin Rev Allergy Immunol. 2014 Dec;47(3):264-73. *

Interesting review focusing to approach the complex group of myositis spectrum diseases as clinicoserological syndromes rather than histopathological subgroups.

12. Lundberg IE, Forbess CJ. Mortality in idiopathic inflammatory myopathies. Clin Exp Rheumatol. 2008; 26(5)(suppl 51):S109-S114. 
13. Rider LG, Lachenbruch PA, Monroe JB, et al; IMACS Group. Damage extent and predictors in adult and juvenile dermatomyositis and polymyositis as determined with the myositis damage index. Arthritis Rheum. 2009;60(11):3425-3435.

14. International myosisitis assessment \& clinical studies group (IMACS): global approaches to improved treatments and understanding of myositis.: National Institute of Environmental Health Sciences; [cited 2015]. Available from: http://www.niehs.nih.gov/research/resources/imacs/index.cfm.

15. Rider LG,Weth VP, Huber AM, et al. Measures of adult and juvenile dermatomyositis, polymyositis, and inclusion body myositis: Physician and Patient/Parent Global Activity, Manual Muscle Testing (MMT), Health Assessment Questionnaire (HAQ)/Childhood Health Assessment Questionnaire (C-HAQ), Childhood Myositis Assessment Scale (CMAS), Myositis Disease Activity Assessment Tool (MDAAT), Disease Activity Score (DAS), Short Form 36 (SF36), Child Health Questionnaire ( $\mathrm{CHQ}$ ), physician global damage, Myositis Damage Index (MDI), Quantitative Muscle Testing (QMT), Myositis Functional Index-2 (FI-2), Myositis Activities Profile (MAP), Inclusion Body Myositis Functional Rating Scale (IBMFRS), Cutaneous Dermatomyositis Disease Area and Severity Index (CDASI), Cutaneous Assessment Tool (CAT), Dermatomyositis Skin Severity Index (DSSI), Skindex, and Dermatology Life Quality Index (DLQI). Arthritis Care Res 2011;63(Suppl. 11):S118-57

16. Aggarval R, Rider LG, Ruperto $\mathrm{N}$ et al. 2016 American College of Rheumatology/European League Against Rheumatism criteria for minimal, moderate, and major clinical response in adult dermatomyositis and polymyositis: An International Myositis Assessment and Clinical Studies Group/Paediatric Rheumatology International Trials Organisation Collaborative Initiative. Ann Rheum Dis. 2017 May;76(5):792-801. **

This criteria set has been approved by the American College of Rheumatology (ACR) Board of Directors and the European League Against Rheumatism (EULAR) Executive Committee.

17. Aggarval R, Rider LG, Ruperto $\mathrm{N}$ et al. 2016 American College of Rheumatology/European League Against Rheumatism Criteria for Minimal, Moderate, and Major Clinical Response in Adult Dermatomyositis and Polymyositis: An International Myositis Assessment and Clinical Studies Group/Paediatric Rheumatology International Trials Organisation Collaborative Initiative. Arthritis Rheumatol. 2017 May;69(5):898-910. **

This criteria set has been approved by the American College of Rheumatology (ACR) Board of Directors and the European League Against Rheumatism (EULAR) Executive Committee.

18. Joffe MM, Love LA, Leff RL, et al. Drug therapy of the idiopathic inflammatory myopathies: predictors of response to prednisone, azathioprine, and methotrexate and a comparison of their efficacy. Am J Med. 1993;94(4):379-387.

19. Bolosiu HD, Man L, Rednic S. The effect of methylprednisolone pulse therapy in polymyositis/dermatomyositis. Adv Exp Med Biol. 1999;455:349-357

20. Hasija R, Pistorio A, Ravelli A, et al. Therapeutic approaches in the treatment of juvenile dermatomyositis in patients with recent-onset disease and in those experiencing disease flare: an international multicenter PRINTO study. Arthritis Rheum 2011; 63:3142-3152. 
21. Hornung $T$, Ko A, Tuting $T$, et al. Efficacy of low-dose methotrexate in the treatment of dermatomyositis skin lesions. Clin Exp Dermatol. 2012 Mar;37(2):139-42. *

22. Combined Treatment of Methotrexate + Glucocorticoids Versus Glucocorticoids Alone in Patients With PM and DM (Prometheus): ClinicalTrials.gov Identifier: NCT00651040

23. Schiopu E, Phillips K, MacDonald PM, et al. Predictors of survival in a cohort of patients with polymyositis and dermatomyositis: effect of corticosteroids, methotrexate and azathioprine. Arthritis Res Ther. 2012 Jan 27;14(1):R22. *

This study from the USA evaluated the predictors of survival in polymyositis/dermatomyositis patients and concluded that younger age, female and Caucasian have longer survival. Also, methotrexate and azathioprine showed similar survival.

24. Yu KH, Wu YJ, Kuo CF, et al. Survival analysis of patients with dermatomyositis and polymyositis: analysis of 192 Chinese cases. Clin Rheumatol 2011; 30:1595-1601. *

A chinese survival analysis study of patients with dermatomyositis showing use of azathioprine had better survival.

25. Wan J, Imadojemu S, Werth VP. Management of rheumatic and autoimmune blistering disease in pregnancy and postpartum. Clin Dermatol. 2016 May-Jun;34(3):344-52.

26. Villalba L, Hicks JE, Adams EM et al. Treatment of refractory myositis: a randomized crossover study of two new cytotoxic regimens. Arthritis Rheum 1998; 41: 392-9.

27. Ge Y, Zhou H, Shi J et al. The efficacy of tacrolimus in patients with refractory dermatomyositis/polymyositis: a systematic review. Clin Rheumatol. 2015 Dec;34(12):2097103. *

This study examined the safety and efficacy of tacrolimus in patients with poly/dermatomyositis and concluded that tacrolimus seems to be a safe drug and well tolerated that improves both muscle strength and lung function.

28. Ueno KI, Shimojima Y, Kishida D, et al. Advantage of administering tacrolimus for improving prognosis of patients with polymyositis and dermatomyositis. Int J Rheum Dis. 2016 Dec;19(12):1322-1330.

29. Vencovsky J, Jarosova K, Machacek S, et al. Cyclosporine A versus methotrexate in the treatment of polymyositis and dermatomyositis. Scand J Rheumatol 2000;29:95-102.

30. Kotani T, Makino S, Takeuchi T, et al. Early intervention with corticosteroids and cyclosporin $A$ and 2-h postdose blood concentration monitoring improves the prognosis of acute/subacute interstitial pneumonia in dermatomyositis. J Rheumatol 2008; 35:254-259.

31. Kotani T, Takeuchi T, Makino S, et al. Combination with corticosteroids and cyclosporin-A improves pulmonary function test results and chest HRCT findings in dermatomyositis patients with acute/subacute interstitial pneumonia. Clin Rheumatol 2011; 30:1021-1028. 
32. Shimojima Y, Ishii W, Matsuda M, et al. Effective Use of Calcineurin Inhibitor in Combination Therapy for Interstitial Lung Disease in Patients With Dermatomyositis and Polymyositis. J Clin Rheumatol. 2017 Mar;23(2):87-93.

33. Yamasaki Y1, Yamada H, Yamasaki M, et al. Intravenous cyclophosphamide therapy for progressive interstitial pneumonia in patients with polymyositis/dermatomyositis. Rheumatology (Oxford). 2007 Jan;46(1):124-30.

34. Riley P, Maillard SM, Wedderburn LR, et al. Intravenous cyclophosphamide pulse therapy in juvenile dermatomyositis. Rheumatology (Oxford). 2004; 43(4):491-496.

35. Dastmalchi M, Notarnicola A, Dani L, et al. Intravenous Cyclophosphamide According to the Euro-Lupus Nephritis Protocol for Progressive Interstitial Lung Disease in Patients with Polymyositis/Dermatomyositis. 2nd Global Conference on Myositis; Abstract no: 337.

36. Rowin J, Amato AA, Deisher N, et al. Mycophenolate mofetil in dermatomyositis: is it safe? Neurology 2006; 66: 1245-7.

37. Morganroth PA, Kreider ME, Werth VP. Mycophenolate mofetil for interstitial lung disease in dermatomyositis. Arthritis Care Res (Hoboken) 2010; 62: 1496-501.

38. Gelber AC, Nousari HC, Wigley FM. Mycophenolate mofetil in the treatment of severe skin manifestations of dermatomyositis: a series of 4 cases. J Rheumatol 2000; 27: 1542-5.

39. Dalakas MC, Illa I, Dambrosia JM, et al. A controlled trial of high-dose intravenous immune globulin infusions as treatment for dermatomyositis. N Engl J Med. 1993;329(27):1993-2000.

This is the only placebo-controlled study showing beneficial effect of IVIG in patients with dermatomyositis

40. Femia AN, Eastham $A B$, Lam $C$, et al. Intravenous immunoglobulin for refractory cutaneous dermatomyositis: a retrospective analysis from an academic medical center. J Am Acad Dermatol 2013;69:654-7.

41. E. Saito, T. Koike, H. Hashimoto, N, et al. Efficacy of high-dose intravenous immunoglobulin therapy in Japanese patients with steroid-resistant polymyositis and dermatomyositis Mod Rheumatol, 18 (2008), pp. 34-44

42. Danieli MG, Calcabrini L, Calabrese V, et al. Intravenous immunoglobulin as add on treatment with mycophenolate mofetil in severe myositis. Autoimmun Rev. 2009;9:124-7.

43. Lam CG, Manlhiot C, Pullenayegum EM, et al. Efficacy of intravenous Ig therapy in juvenile dermatomyositis. Ann Rheum Dis 2011; 70:2089-2094.

44. Danieli MG, Pettinari L, Moretti R, et al. Subcutaneous immunoglobulin in polymyositis and dermatomyositis: a novel application. Autoimmun Rev. 2011 Jan;10(3):144-9. *

This study retrospectively describe seven myositis patients treated with subcutaneous immunoglobulin 
45. Danieli MG, Moretti R, Gambini S, et al.: Open-label study on treatment with $20 \%$ subcutaneous IgG administration in polymyositis and dermatomyositis. Clin Rheumatol. 2014 Apr;33(4):531-6.

46. Chiu YE, Co DO. Juvenile dermatomyositis: immunopathogenesis, role of myositis-specific autoantibodies, and review of rituximab use [published erratum appears in Pediatr Dermatol 2011; 28:627]. Pediatr Dermatol 2011;28:357-67.

47. Rios Fernandez R, Callejas Rubio JL, Sanchez Cano D, et al. Rituximab in the treatment of dermatomyositis and other inflammatory myopathies. A report of 4 cases and review of the literature. Clin Exp Rheumatol 2009; 27:1009-1016.

48. Levine TD: Rituximab in the treatment of dermatomyositis: an open-label pilot study. Arthritis Rheum. 2005 Feb;52(2):601-7.

49. Oddis CV, Reed AM, Aggarwal R et al. RIM Study Group. Rituximab in the treatment of refractory adult and juvenile dermatomyositis and adult polymyositis: a randomized, placebo-phase trial. Arthritis Rheum. 2013 Feb;65(2):314-24.

The largest randomized study with refractory inflammatory myopathies using the IMACS tools. The final results did not show difference in the time to improvement, but $83 \%$ of the patients met the DOI after RTX administration.

50. Aggarwal R, Bandos A, Reed AM, et al. Predictors of clinical improvement in rituximabtreated refractory adult and juvenile dermatomyositis and adult polymyositis. Arthritis Rheumatol 2014;66(3):740-9.

Subanalysis of the RIM study, showing that the presence of an antisynthetase, primarily antiJo-1, anti-Mi-2, or other autoantibody predicted a shorter time to improvement compared to the absence of autoantibodies.

51. Aggarwal R, Oddis CV, Goudeau D, et al. Autoantibody levels in myositis patients correlate with clinical response during B cell depletion with rituximab. Rheumatology (Oxford). 2016 Jun;55(6):991-9.

52. Arabshahi B, Silverman RA, Jones OY, et al. Abatacept and sodium thiosulfate for treatment of recalcitrant juvenile dermatomyositis complicated by ulceration and calcinosis. J Pediatr 2012; 160:520-522

53. Lundberg IE, Tjärnlund A, Tang Q, et al. Abatacept in the Treatment of Adult Dermatomyositis and Polymyositis: a Randomized, Treatment Delayed-Start Trial [abstract]. Arthritis Rheumatol. 2015; 67 (suppl 10). http://acrabstracts.org/abstract/abatacept-in-thetreatment-of-adult-dermatomyositis-and-polymyositis-a-randomized-treatment-delayedstart-trial/.

54. DeBleeker JL, Meire VI, Declercq W, et al. Immunolocalization of tumor necrosis factor-alpha and its receptors in inflammatory myopathies. Neuromusc Dis 1999;9:239-246. 
55. Muscle Study Group. A randomized, pilot trial of etanercept in dermatomyositis. Ann Neurol 2011; 70:427-436.

56. Rouster-Stevens KA, Ferguson L, Morgan G, et al. Pilot study of etanercept in patients with refractory juvenile dermatomyositis. Arthritis Care Res (Hoboken). 2014 May;66(5):783-7.

57. Klein R, Rosenbach $M$, Kim EJ, et al. Tumor necrosis factor inhibitor-associated dermatomyositis. Arch Dermatol. 2010 Jul;146(7):780-4

58. Dicaro D, Bowen C, Dalton SR. Dermatomyositis associated with anti-tumor necrosis factor therapy in a patient with psoriasis. J Am Acad Dermatol. 2014 Mar;70(3):e64-5.

59. Quain RD, Werth VP.: Management of cutaneous dermatomyositis: current therapeutic options. Am J Clin Dermatol. 2006;7(6):341-51.

60. Sontheimer RD: The management of dermatomyositis: current treatment options. Expert Opin Pharmacother. 2004 May;5(5):1083-99.

61. Ueda $M$, Makinodan $R$, Matsumum $M$, et al. Successful treatment of amyopathic dermatomyositis with topical tacrolimus. Br J Dermatol 2003; 148: 595-6.

62. Hollar CB, Jorizzo JL. Topical tacrolimus $0.1 \%$ ointment for refractory skin disease in dermatomyositis: a pilot study. J Dermatol Treat 2004; 15: 35-9.

63. Kim JE, Jeong MG, Lee $\mathrm{HE}$, et al. Successful treatment of cutaneous lesions of dermatomyositis with topical pimecrolimus. Ann Dermatol. 2011 Aug;23(3):348-51.

64. Betteridge Z, McHugh N: Myositis-specific autoantibodies: an important tool to support diagnosis of myositis. J Intern Med. 2016 Jul;280(1):8-23. **

This review describe the main myositis-specific and myositis-associated autoantibodies, their frequencies and clinical associations across different ages and ethnic groups.

65. Harsha Gunawardena, Zoe E. Betteridge and Neil J. McHugh: Myositis-specific autoantibodies: their clinical and pathogenic significance in disease expression. Rheumatology 2009;48:607-612

66. Lega JC, Fabien N, Reynaud Q, et al. The clinical phenotype associated with myositis-specific and associated autoantibodies: A meta-analysis revisiting the so-called antisynthetase syndrome. Autoimmun Rev., 2014 Sep; 13(9):883-91. **

A meta-analysis showing Forest plot of clinical features and outcomes associated with antiJo1 and non-anti-Jo1 autoantibodies.

67. Rojas-Serrano J, Herrera-Bringas D, Mejía M, et al. Prognostic factors in a cohort of antisynthetase syndrome (ASS): serologic profile is associated with mortality in patients with interstitial lung disease (ILD). Clin Rheumatol (2015) 34:1563-1569 
68. Aggarwal $\mathrm{R}$, Cassidy $\mathrm{E}$, Fertig $\mathrm{N}$ et al. Patients with non-Jo-1 anti-tRNA-synthetase autoantibodies have worse survival than Jo-1 positive patients. Ann Rheum Dis 2014; 73: 227-32. *

Survival of 202 patients with antisynthetase syndrome was reviewed retrospectively showing Non-Jo-1 anti-synAb positive patients have decreased survival compared with Jo-1 patients.

69. Johnson C, Pinal-Fernandez I, Parikh R, et al. Assessment of Mortality in Autoimmune Myositis With and Without Associated Interstitial Lung Disease. Lung. 2016 Oct;194(5):733-7.

70. Váncsa A, Csípo I, Németh J, et al. Characteristics of interstitial lung disease in SS-A positive/Jo-1 positive inflammatory myopathy patients. Rheumatol Int. 2009 Jul;29(9):98994.

71. Sharp C, McCabe M, Dodds N, et al. Rituximab in autoimmune connective tissue diseaseassociated interstitial lung disease. Rheumatology (Oxford). 2016 Jul;55(7):1318-24.

72. Bauhammer J, Blank N, Max R, et al. Rituximab in the Treatment of Jo1 Antibody-associated Antisynthetase Syndrome: Anti-Ro52 Positivity as a Marker for Severity and Treatment Response. J Rheumatol. 2016 Aug;43(8):1566-74.

73. Tansley SL, Betteridge ZE, McHugh NJ. The diagnostic utility of autoantibodies in adult and juvenile myositis. Curr Opin Rheumatol 2013;25:772-7.

74. Targoff IN, Reichlin M. The association between Mi-2 antibodies and dermatomyositis. Arthritis Rheum 1985; 28: 796-803.

75. Ghirardello A, Zampieri S, laccarino L et al. Anti-Mi-2 antibodies. Autoimmunity 2005; 38: 7983.

76. Ghirardello A, Borella E, Beggio M, et al. Myositis autoantibodies and clinical phenotypes. Auto Immun Highlights 2014;5(3):69-75.

77. Targoff IN, Mamyrova G, Trieu EP, et al. A novel autoantibody to a $155-\mathrm{kd}$ protein is associated with dermatomyositis. Arthritis Rheum. 2006 Nov;54(11):3682-9.

78. Trallero-Araguas E, Rodrigo-Pendas JA, Selva-O'Callaghan A, et al. Usefulness of anti-p155 autoantibody for diagnosing cancer-associated dermatomyositis: a systematic review and meta-analysis. Arthritis Rheum 2012; 64(2):523-32.

79. Valenzuela A, Chung L, Casciola-Rosen L, et al. Identification of clinical features and autoantibodies associated with calcinosis in dermatomyositis. JAMA Dermatol. 2014 Jul;150(7):724-9.

80. Sato S, Hirakata M, Kuwana M et al. Autoantibodies to a 140-kd polypeptide, CADM-140, in Japanese patients with clinically amyopathic dermatomyositis. Arthritis Rheum 2005; 52: 1571-6. 
81. Chen Z, Hu W, Wang Y, et al. Distinct profiles of myositis-specific autoantibodies in Chinese and Japanese patients with polymyositis/dermatomyositis. Clin Rheumatol. 2015 Sep;34(9):1627-31

82. Hall JC, Casciola-Rosen L, Samedy LA et al. Anti-melanoma differentiation-associated protein 5-associated dermatomyositis: expanding the clinical spectrum. Arthritis Care Res (Hoboken). 2013 Aug;65(8):1307-15.

83. Kawasumi H, Gono $\mathrm{T}$, Kawaguchi $\mathrm{Y}$, et al. Recent Treatment of Interstitial Lung Disease with Idiopathic Inflammatory Myopathies. Clin Med Insights Circ Respir Pulm Med. 2015; 9(Suppl 1): $9-17 . *$

This manuscript provide a useful therapeutic strategy for ILDs associated with PM/DM based on clinical course. antibody type, ferritin level and response to treatment.

84. Yamasaki Y, Yamada H, Ohkubo $\mathrm{M}$, et al. Long-term survival and associated risk factors in patients with adult-onset idiopathic inflammatory myopathies and amyopathic dermatomyositis: experience in a single institute in Japan. J Rheumatol. 2011;38(8):1636-43.

85. Betteridge ZE, Gunawardena H, Chinoy $\mathrm{H}$ et al. Clinical and human leucocyte antigen class II haplotype associations of autoantibodies to small ubiquitin-like modifier enzyme, a dermatomyositis-specific autoantigen target, in UK Caucasian adult-onset myositis. Ann Rheum Dis 2009; 68: 1621-5.

86. Bodoki L, Nagy-Vincze M, Griger Z, et al. Four dermatomyositis-specific autoantibodiesantiTIF1gamma, anti-NXP2, anti-SAE and anti-MDA5-in adult and juvenile patients with idiopathic inflammatory myopathies in a Hungarian cohort. Autoimmun Rev 2014; 13: 1211-9.

87. Tarricone E, Ghirardello A, Rampudda M, et al. Anti-SAE antibodies in autoimmune myositis identification by unlabelled protein immunoprecipitation in an Italian patient cohort. J Immunol Methods 2012;384(1):128-34.

88. Gunawardena H, Wedderburn LR, Chinoy H et al. Autoantibodies to a $140-k d$ protein in juvenile dermatomyositis are associated with calcinosis. Arthritis Rheum 2009; 60: 1807-14.

89. Ichimura Y, Matsushita T, Hamaguchi Y et al. Anti-NXP2 autoantibodies in adult patients with idiopathic inflammatory myopathies: possible association with malignancy. Ann Rheum Dis 2012; 71: 710-3.

90. Fiorentino DF, Chung LS, Christopher-Stine L et al. Most patients with cancer-associated dermatomyositis have antibodies to nuclear matrix protein NXP-2 or transcription intermediary factor 1gamma. Arthritis Rheum 2013; 65: 2954-62.*

Analyzed patient sera from DM cohorts $(n=213)$ showed that reactivity against either NXP-2 or TIF- $1 \gamma$ identified $83 \%$ of cancer-associated DM.

91. Fredi M, Bartoli F, Cavazzana I, et al. Calcinosis in poly-dermatomyositis: clinical and laboratory predictors and treatment options. Clin Exp Rheumatol. 2017 Mar-Apr;35(2):303308. 
92. Mammen AL, Chung T, Christopher-Stine L, et al. Autoantibodies against 3-hydroxy-3methylglutaryl-coenzyme A reductase in patients with statin-associated autoimmune myopathy. Arthritis Rheum. 2011; 63: 713-21.

93. Takayuki Kishi, Lisa G. Rider, Katherine Pak, et al. Anti-3-Hydroxy-3-Methylglutaryl-Coenzyme A Reductase Autoantibodies are Associated with DRB1*07:01 and Severe Myositis in Pediatric Myositis Patients. Arthritis Care Res (Hoboken). 2017 Jul;69(7):1088-1094.

94. Tansley SL, Betteridge ZE, Simou S et al. Anti-HMGCR Autoantibodies in Juvenile Idiopathic Inflammatory Myopathies Identify a Rare but Clinically Important Subset of Patients. J Rheumatol. 2017 Apr;44(4):488-492.

95. Rider LG, Dankó K, Miller FW.: Myositis registries and biorepositories: powerful tools to advance clinical, epidemiologic and pathogenic research. Curr Opin Rheumatol. 2014 Nov;26(6):724-41.

96. Euromyositis: International collaboration research and treatment database for myositis specialists. Available: https://euromyositis.eu/ 